\title{
Successful treatment of propofol-related infusion syndrome in critically ill patient receiving low-dose propofol infusion: a case report
}

\author{
Nahyeon Park, Tae Sun Ha \\ Department of Surgery, Soonchunhyang University Bucheon Hospital, Soonchunhyang University College of Medicine, Bucheon, Korea
}

Propofol is widely used to sedate agitated patients in intensive care units. However, it can cause a rare but fatal complication, propofol-related infusion syndrome (PRIS). The pathophysiology of PRIS is not clear, and there is no definitive diagnosis and treatment. We report a successfully treated case of PRIS in a critically ill patient receiving low-dose propofol infusion. A 59-year-old male patient complaining of sudden chest pain repeatedly collapsed in an ambulance and the emergency room, and veno-arterial extracorporeal membrane oxygenation was delivered. He was diagnosed with a total occluded left anterior descending coronary artery in coronary angiography. On day 20, he showed arrhythmia, increased creatinine kinase (CK), and increased CK-MB and troponin I, accompanied by unstable hemodynamic status despite high-dose vasopressors. He was administered propofol for 20 days at an average dose of $1.3 \mathrm{mg} / \mathrm{kg} / \mathrm{hr}$ owing to issues with agitation and ventilator synchrony. We strongly suspected PRIS and immediately discontinued propofol infusion, and he was successfully treated with aggressive supportive care. PRIS can occur in patients administered propofol for a prolonged period at low doses. Thus, clinicians should use propofol with caution for PRIS and change to alternative sedatives for long-term sedation.

Key Words: carnitine; extracorporeal membrane oxygenation; propofol; propofol infusion syndrome

Propofol is widely used for the intravenous induction of general anesthesia and other procedures in the operating room, as well as the sedation of agitated patients in intensive care units (ICUs). However, propofol causes various side effects, including hypotension, apnea, sinus bradycardia, depression of cardiac contractility, and rare but fatal propofol-related infusion syndrome (PRIS) [1]. The pathophysiology of PRIS is not clear, and there is no definitive diagnosis and treatment. Herein, we report the successful treatment of PRIS in a patient receiving low-dose propofol infusion with a history of extracorporeal membrane oxygenation (ECMO) for cardiac arrest.

\section{CASE REPORT}

A previously healthy 59-year-old male patient complaining of sudden chest pain collapsed in the ambulance on the way to the emergency room. Emergency rescue technicians per-

\section{Case Report}

Received: June 21, 2021

Revised: August 6, 2021

Accepted: August 24, 2021

Corresponding author

Tae Sun Ha

Department of Surgery,

Soonchunhyang University Bucheon

Hospital, 170 Jomaru-ro, Wonmi-gu,

Bucheon 14584, Korea

Tel: +82-32-621-6265

Fax: +82-32-621-5016

Email:applips@gmail.com

Copyright @ 2023 The Korean Society of Critical Care Medicine

This is an Open Access article distributed under the terms of Creative Attributions Non-Commercial License (https://creativecommons.org/ li-censes/(by-nc/4.0/) which permits unrestricted noncommercial use, distribution, and reproduction in any medium, provided the original work is properly cited. 
formed 20 seconds of cardiopulmonary resuscitation after shocking for ventricular fibrillation, and the return of spontaneous circulation was achieved. Upon arrival at our hospital, his blood pressure was $105 / 78 \mathrm{~mm} \mathrm{Hg}$, heart rate was 120 beats/min, respiratory rate was 20 breaths/min, and body temperature was $35.8^{\circ} \mathrm{C}$. He was diagnosed with ST-segment elevation myocardial infarction on an electrocardiogram (ECG). During the preparation for coronary angiography, repeated cardiac arrest occurred. Thus, we immediately applied veno-arterial (VA) ECMO. Percutaneous coronary intervention reperfused the total occluded left anterior descending coronary artery via thrombectomy. After admission to ICU, he was sedated using remifentanil $(0.2 \mu \mathrm{g} / \mathrm{kg} / \mathrm{min})$ and propofol (FreSOfol MCT 2\%, $1.3 \mathrm{mg} / \mathrm{kg} / \mathrm{hr}$ ). On day 3, his hemodynamic status and oxygenation improved, allowing for VA ECMO decannulation.

On day 5 , he presented with progressive acute kidney injury, so we applied continuous renal replacement therapy (CRRT). Three days after decannulation, we applied veno-venous (VV) ECMO due to severe acute respiratory distress syndrome following hospital-acquired pneumonia. On day 16 , since he showed clinical improvement of pneumonia, we weaned him off VV ECMO. Sedatives for ventilator care were retained without dose reduction due to agitation and ventilator dyssynchrony, but vasopressors were slowly tapered according to improved cardiac function. On day 18, the alanine aminotransferase and aspartate aminotransferase, which had been less than 150 units/L, were abruptly increased to
719 units/L and 211 units/L, respectively and were consistently increased until day 27 (Table 1). On day 20, he showed bundle branch block, increased creatinine kinase (CK), and increased CK-MB and troponin I. Due to suddenly aggravated hypotension, we increased the infusion rate of norepinephrine and epinephrine and added vasopressin. Acute renal failure (ARF) and metabolic acidosis are common features of PRIS, but they were attenuated to the previously maintained CRRT in our case. For 20 days, he received propofol administration at an average dose of $1.3 \mathrm{mg} / \mathrm{kg} / \mathrm{hr}$, and we could not decrease the propofol infusion rate owing to issues with agitation and ventilator synchrony. We strongly suspected PRIS and immediately discontinued propofol infusion. The initial serum triglyceride level after admission was $369 \mathrm{mg} / \mathrm{dl}$, but no follow-up monitoring for serum triglyceride levels was performed. The cortisol level and thyroid function tests were within the normal ranges. After 24 hours of discontinuing propofol, the requirement for vasopressors was slowly decreased and vasopressin was tapered according to his stabilized vital signs. However, the CK level continued to elevate to 139,066 IU/L (Table 1). Thus, for recovery from PRIS, we administrated L-carnitine, which mitigates the effects of PRIS by supporting free fatty acid (FFA) metabolism, at a dose of $500 \mathrm{mg}$ three times per day starting on day 23. After 2 days of carnitine administration, the CKlevel gradually declined, and the patient was stabilized with only low-dose norepinephrine $(0.05 \mu \mathrm{g} /$ $\mathrm{kg} / \mathrm{min}$ ). He was discharged from the ICU on day 51 and transferred to a nursing hospital for rehabilitation on day 92 .

Table 1. Laboratory data in the intensive care unit

\begin{tabular}{lccccccccccc}
\hline Variable & Reference range & HD 16 & HD 18 & HD 20 & HD 21 & HD 22 & HD 23 & HD 24 & HD 26 & HD 28 & HD 30 \\
\hline CK-MB (ng/mL) & 0.1 to 6.3 & 4.8 & 18.4 & 61.0 & 105.0 & 210.0 & 303.0 & 395.0 & 140.0 & 23.8 & 12.6 \\
Troponin I (ng/L) & 0.00 to 11.3 & 3.6 & 5.0 & 4.9 & 4.6 & 4.8 & 5.0 & 3.7 & 2.2 & 1.4 & 0.6 \\
CK (U/L) & 20 to 171 & - & - & 18,063 & 27,264 & 62,250 & 139,066 & 178,730 & 46,330 & 2,531 & 77 \\
Lactic acid (mmo/L) & 0.5 to 2.0 & 0.9 & 0.5 & 1.0 & 1.9 & 2.2 & 3.5 & 4.0 & 2.6 & 2.1 & 1.8 \\
$\mathrm{AST}(\mathrm{U} / \mathrm{L})$ & 5 to 45 & 131 & 719 & 1,110 & 1,414 & 2,145 & 3,090 & 3,375 & 1,333 & 345 & 170 \\
$\mathrm{ALT}(\mathrm{U} / \mathrm{L})$ & 0 to 40 & 79 & 211 & 272 & 303 & 542 & 771 & 1,135 & 805 & 439 & 213 \\
$\mathrm{PH}$ & 7.35 to 7.45 & 7.43 & 7.33 & 7.41 & 7.41 & 7.53 & 7.46 & 7.43 & 7.48 & 7.44 & 7.49 \\
$\mathrm{PaCO}(\mathrm{mm} \mathrm{Hg})$ & 35 to 45 & 30 & 46 & 37 & 32 & 25 & 29 & 36 & 32 & 36 & 29 \\
$\mathrm{PaO}_{2}(\mathrm{~mm} \mathrm{Hg})$ & 75 to 100 & 107 & 124 & 149 & 92 & 97 & 93 & 101 & 94 & 80 & 99 \\
$\mathrm{HCO}_{3}^{-}(\mathrm{mmol} / \mathrm{L})$ & 23 to 29 & 25.0 & 23.7 & 22.7 & 20.3 & 20.6 & 20.1 & 23.9 & 23.6 & 24.5 & 21.6 \\
$\mathrm{BE}_{(\mathrm{mmol} / \mathrm{L})}$ & -2 to 2 & 0.7 & -2.3 & -1.6 & -3.4 & -0.9 & -2.8 & -0.9 & 0.5 & 0.6 & -0.9 \\
$\mathrm{Na}^{+}(\mathrm{mmol} / \mathrm{L})$ & 136 to 145 & 137 & 133 & 132 & 133 & 135 & 132 & 132 & 137 & 138 & 136 \\
$\mathrm{~K}^{+}(\mathrm{mmol} / \mathrm{L})$ & 3.5 to 5.5 & 3.6 & 4.1 & 3.9 & 3.6 & 4.1 & 4.5 & 4.5 & 3.7 & 3.5 & 3.6 \\
\hline
\end{tabular}

Continuous renal replacement therapy is started on HD 5 .

HD: hospital day; CK: creatinine kinase; AST; aspartate aminotransferase; $\mathrm{ALT}$ : alanine aminotransferase; $\mathrm{PaCO}_{2}$ : partial pressure of carbon dioxide; PaO ${ }_{2}$ : arterial partial pressure of oxygen; $\mathrm{HCO}_{3}^{-}$: bicarbonate; $\mathrm{BE}$ : base excess; $\mathrm{Na}^{+}$: sodium; $\mathrm{K}^{+}$: potassium. 


\section{DISCUSSION}

PRIS is a rare but lethal syndrome with an incidence of $1.1 \%$ and a mortality of $18 \%$ [2]. The current definition of PRIS includes metabolic acidosis, lactic acidosis, rhabdomyolysis, hyperkalemia, renal failure, hyperlipidemia, Brugada-like ECG change, arrhythmia, and cardiac failure. As most studies have shown, PRIS generally occurs in critically ill patients receiving propofol at a high dose $(>5 \mathrm{mg} / \mathrm{kg} / \mathrm{hr}$ ) and prolonged ( $>48$ hours) administration [2-5]. However, in our case, he had received a low-dose propofol infusion rate of $1.3 \mathrm{mg} / \mathrm{kg} / \mathrm{hr}$, which is currently regarded as a safe dose, for sedation in the ICU. Although the previous implementation of CRRT masked metabolic acidosis and hyperkalemia, our patient showed typical PRIS symptoms on day 20, including lactic acidosis, rhabdomyolysis, arrhythmia, and unstable hemodynamics. Recently, a few studies reported PRIS cases after propofol infusion from low-dose or short duration in non-critically ill patients [6]. Since these cases showed only a subset of PRIS symptoms with metabolic acidosis, unlike our case, it is uncertain whether these reports represent actual manifestations of PRIS.

The pathophysiology of PRIS is still unknown due to the complicated process. Recent clinical and experimental studies have focused on dysfunction of the mitochondrial respiratory chain caused by propofol. Propofol inhibits carnitine palmitoyltransferase I, an outer membrane mitochondrial protein that transfers long-chain FFA into the mitochondria [7]. As a consequence of inhibiting the beta-oxidation of fatty acids, the accumulation of unutilized fatty acids is increased in diverse organs, and the production of adenosine triphosphate (ATP) is decreased in the mitochondrial respiratory chain. Also, propofol directly disturbs the flow of electrons down the mitochondrial respiratory chain by interacting with coenzyme $\mathrm{Q}$ [8]. Under stress conditions, the imbalance between increased energy demand and decreased energy availability results in the depletion of ATP and cellular hypoxia in tissue, especially, in the heart and skeletal muscles. Lastly, propofol acts as an antagonist at beta-adrenoceptors and calcium channels in the heart, leading to decreased cardiac function and resistance to inotropes [9]. Because the occurrence of PRIS appears to result from an interaction between the pharmacologic action of propofol and clinical host conditions, it is important to know the risk factors associated with PRIS. In addition to high dose of propofol for a prolonged period, the cumulative dose of propofol higher than $240 \mathrm{mg}$ is a significant risk factor for PRIS development [5]. Other potential other predisposing factors are the use of catecholamine and glucocorticoid, carbohydrate exhaustion, carnitine deficiency, inborn impairment of fatty acid oxidation, and critical illnesses including sepsis and neurological injuries [10]. In critically ill condition, catecholamines, which is activated by the central nervous system, and glucocorticoids contribute to acute skeletal and cardiac muscle damage as priming factors. Propofol administration also functions as a triggering factor that causes energy deprivation and imbalance by mitochondria dysfunction, which lead to myocytolysis in skeletal and cardiac muscles. In our case, although our patient received a low dose propofol infusion, the cumulative propofol dose was high as $624 \mathrm{mg} / \mathrm{kg}$, and he has received a high dose of vasopressors at the time.

The clinical features of PRIS include a sudden increase in lactic acid, unexplained metabolic acidosis, elevated CK and myoglobin, hyperlipidemia, and ECG changes. However, it is challenging to recognize PRIS symptoms in the early stages because its symptoms are nonspecific and overlap with signs that will commonly occur in critically ill patients with shock and organ dysfunctions. Especially, metabolic acidosis and rhabdomyolysis generally manifest in the early stage of PRIS, but these symptoms may be masked in a patient receiving CRRT for ARF, thereby delaying the diagnosis of PRIS [5]. Furthermore, because the sedatives in ECMO patients need to reduce the risk of catheter dislodgement, to relieve pain, anxiety, and distress from critical illness, and to facilitate synchronization between ventilator and patient, it is commonly used high doses for safety, consequently, may increase the risk for the PRIS development. Thus, clinicians should use propofol with caution for PRIS in critically ill patients who need long-term sedation, especially those undergoing CRRT or ECMO.

The initiation of successful treatment depends upon the early recognition of PRIS and the immediate discontinuation of propofol administration with a change to alternative sedative agents. The treatment for PRIS is aimed at the clinical manifestation of PRIS because there are no established or antidote treatment guidelines for PRIS. CRRT should be considered in patients showing persistent metabolic acidosis despite the administration of sodium bicarbonate. Vasopressors and inotropic agents will be needed in patients with cardiogenic shock, and especially, ECMO should be considered for patients with refractory cardiogenic shock. As L-carnitine can transfer longchain fatty acids to the mitochondria for beta-oxidation, adequate L-carnitine supply may support FFA metabolism [11]. Moriyama et al. [12] showed that the administration of L-car- 
nitine in an animal study could facilitate FFA metabolism and prevent elevations in FFA concentrations induced by propofol. In our case, we used L-carnitine after recognizing PRIS because we thought that supplementation with L-carnitine was unlikely to cause harm and may be beneficial considering the etiology of PRIS. It is difficult to assess how much L-carnitine significantly affected the improvement of the patient in this case with PRIS. However, our patient showed a rapid recovery after L-carnitine supplementation.

The best management of PRIS is prevention rather than treating PRIS that has occurred. Since the risk of PRIS is related to the dose and duration of propofol, it is recommended that the administration of propofol is used for less than 48 hours and less than the dose of $5 \mathrm{mg} / \mathrm{kg} / \mathrm{hr}$. If propofol sedation will be prolonged or a high dose cannot be avoided, such as applied ECMO, shock, and neurological injuries, clinicians should use propofol in as small a dose as possible by using a multimodal sedation regimen and change to alternative sedatives if the patient receives propofol at high dose $(>5 \mathrm{mg} / \mathrm{kg} / \mathrm{hr})$ or prolonged ( $>48$ hours) or high cumulative doses ( $>240 \mathrm{mg}$ / $\mathrm{kg}$ ). Clinicians should routinely monitor $\mathrm{PH}$, lactate, triglyceride, CK, and cardiac marker, and should particularly check CK daily in a patient receiving CRRT to prevent delayed PRIS recognition due to masked metabolic acidosis by CRRT. Because the depletion of carbohydrates can increase lipolysis, early adequate supplementation with carbohydrates while minimizing lipids can prevent PRIS. In this case, we overlooked the PRIS risk that could have occurred by long-term administration because propofol was administered at a low dose within the recommended dose range.

In conclusion, PRIS can occur in patients administered propofol for a prolonged period at low doses that are currently considered safe, as well as at high doses. Thus, clinicians should use propofol for a short period with a high index of suspicion for PRIS and change to alternative sedatives for longterm sedation. Although the role of L-carnitine for PRIS is uncertain, we propose considering L-carnitine supplementation based on the favorable outcome in our case, and further studies are needed to evaluate the effect of L-carnitine for treating PRIS.

\section{CONFLICT OF INTEREST}

No potential conflict of interest relevant to this article was reported.

\section{FUNDING}

None.

\section{ACKNOWLEDGMENTS}

None.

\section{ORCID}

Nahyeon Park https://orcid.org/0000-0003-4773-0361

Tae Sun Ha https://orcid.org/0000-0003-3683-6929

\section{AUTHOR CONTRIBUTIONS}

Conceptualization: TSH. Data curation: NHP. Formal analysis: NHP. Methodology: NHP. Project administration: TSH. writing-original draft: NHP. Writing-review \& editing: TSH.

\section{REFERENCES}

1. Hatch DJ. Propofol-infusion syndrome in children. Lancet 1999;353:1117-8.

2. Roberts RJ, Barletta JF, Fong JJ, Schumaker G, Kuper PJ, Papadopoulos S, et al. Incidence of propofol-related infusion syndrome in critically ill adults: a prospective, multicenter study. Crit Care 2009;13:R169.

3. Cremer OL, Moons KG, Bouman EA, Kruijswijk JE, de Smet AM, Kalkman CJ. Long-term propofol infusion and cardiac failure in adult head-injured patients. Lancet 2001;357:117-8.

4. Mirrakhimov AE, Voore P, Halytskyy O, Khan M, Ali AM. Propofol infusion syndrome in adults: a clinical update. Crit Care Res Pract 2015;2015:260385.

5. Hemphill S, McMenamin L, Bellamy MC, Hopkins PM. Propofol infusion syndrome: a structured literature review and analysis of published case reports. Br J Anaesth 2019;122:448-59.

6. Fodale V, La Monaca E. Propofol infusion syndrome: an overview of a perplexing disease. Drug Saf 2008;31:293-303.

7. Wolf A, Weir P, Segar P, Stone J, Shield J. Impaired fatty acid oxidation in propofol infusion syndrome. Lancet 2001;357:606-7.

8. Schenkman KA, Yan S. Propofol impairment of mitochondrial respiration in isolated perfused guinea pig hearts determined by reflectance spectroscopy. Crit Care Med 2000;28:172-7.

9. Zhou W, Fontenot HJ, Liu S, Kennedy RH. Modulation of cardiac calcium channels by propofol. Anesthesiology 1997;86:670-5. 10. Park SY, Kim EG, Park HP. Propofol-related infusion syndrome 
in an adult patient using propofol coma therapy to control intracranial pressure. Korean J Crit Care Med 2013;28:234-8.

11. Uezono S, Hotta Y, Takakuwa Y, Ozaki M. Acquired carnitine deficiency: a clinical model for propofol infusion syndrome? Anesthesiology 2005;103:909.
12. Moriyama T, Kiyonaga N, Ushikai M, Kawaguchi H, Horiuchi M, Kanmura Y. Effects of L-carnitine on propofol-induced inhibition of free fatty acid metabolism in fasted rats and in vitro. Open J Anesthesiol 2018;8:147-58. 